\title{
A note on Merwin's measurements of forward flow in rolling contact
}

\author{
A.R.S. Ponter ${ }^{a}$, L. Afferrante ${ }^{b}$, M. Ciavarella ${ }^{b, *}$ \\ a Department of Engineering, University of Leicester, University Road, Leicester LE1 7RH, UK \\ b Dipartimento di Ingegneria Meccanica e Gestionale (DIMeG), Centro di Eccellenza in Meccanica Computazionale (CEMeC), \\ V. le Japigia 182, Politecnico di Bari, 70125 Bari, Italy
}

Received 12 November 2002; received in revised form 9 May 2003; accepted 19 May 2003

\begin{abstract}
The first quantitative analysis of the forward flow in frictionless rolling contact, firstly discovered experimentally by Crook [Proc. Inst. Mech. Eng. London 171 (1957) 187], was conducted by Merwin [Plastic deformation of surfaces in rolling, Ph.D. Dissertation, Cambridge University, UK, 1962] who attempted to model the ratchetting phenomenon in excess of shakedown (the cumulative forward flow due to continuous shear strain increase observed in experiments) as a function of load using a simple perfect plasticity model and a simplified solution to the elasto-plastic problem. However, later FEM analysis [J. Appl. Mech., Trans. ASME 52 (1985) 67, 75] and more refined calculations still based on perfect plasticity but using distributed dislocations [J. Mech. Phys. Solids 33 (1987) 61], found that the ratchet rate was much higher than what measured in experiments, showing the Merwin's approximate solution method was not effective. However, later analysis have concentrated on sophisticated non-linear hardening laws, also because the ratchetting strain rate was found to slowly decay in rail steel materials. This note is focused on another, less known, aspect of the original Merwin's analysis: his material data were limited to monotonic curves, but his yield limit choice corresponds for around $1 \%$ for mild steel and Dural, but to nearly $25 \%$ deformation in copper, indicating that hardening plays a significant role into the mechanics of the problem, and that Merwin had taken this into account a posteriori by looking at the load where ratchetting begins.

The paper suggests that the cyclic strain growth can be divided into two sequential phenomena: the first, assuming there is no long term material ratchetting (MR), i.e. a calculation based upon elastic properties and a monotonic stress-plastic strain curve, and a second, steady state, for a hardened structure, depending only on MR. In the first phase, we assume the plastic flow is dominated by structural ratchetting (SR), i.e. assuming the ratchetting is well described by the perfectly plastic prediction, where the yield limit is increased according to the level of deformation. This process leads to a quick saturation and the following deformation is attributed to the steady-state material response which we denominate MR. Further, it is shown that experimental measurements of Merwin have more to do with MR than SR.
\end{abstract} (C) 2003 Elsevier B.V. All rights reserved.

Keywords: Contact fatigue; Plasticity; Wear; Ratchetting

\section{Introduction}

Fatigue damage in rail-wheel contact and rolling contact fatigue (RCF) has been studied for many years, but with respect to standard fatigue, RCF has progressed more slowly because there are intrinsic difficulties: a highly compressive state of stress is introduced, large plastic deformations are present which are not easy to compute, and standard uniaxial data (both for the plastic response and the fatigue response) are not immediate to use. Additional factors are uncertainties on loads and load spectra induced in real contact of rail-wheel assemblies in service, the interaction with wear and with other tribological factors. Finally, on a more macroscopic scale, railway industry has no comparable ex-

\footnotetext{
* Corresponding author. Tel.: +39-080-5481265.

E-mail address: m.ciavarella@area.ba.cnr.it (M. Ciavarella).
}

tensive testing traditions on a large number of prototypes as car or, even more, aircraft manufacturers [5].

One aspect on which much effort has been devoted is to understand the plastic mechanisms of deformation for the very high pressure sustained by the rail-wheel assembly. Peak pressures of the order of $1 \mathrm{GPa}$ or higher are currently encountered, so that not only the elastic limit is passed, but actually also the elastic shakedown and plastic shakedown. Classical experiments on twin-disk rigs such as Crook [1], Merwin [2] (see also [6]), and Hamilton [7], typically used "soft" materials such as copper. This choice was probably suggested to amplify (and therefore make more easily measurements) plastic deformations, but at the same time copper has a quite marked cyclic hardening behaviour and the mechanism of ratchetting in this material is very different from that of rail steel, ultimately more important for railway industry. In any case, in copper, rolling contact experiments 


\begin{tabular}{|ll|}
\hline \multicolumn{2}{|l|}{ Nomenclature } \\
$a$ & contact area semi-width \\
$G$ & shear modulus \\
$k_{0}$ & yield stress \\
$N$ & number of cycles \\
$p_{0}$ & peak Hertzian pressure \\
& \\
Greek & letters \\
$\gamma$ & ratchetting shear strains \\
$\delta$ & forward flow displacement \\
\hline
\end{tabular}

have generally shown a forward flow which seemed to reach a steady state. Merwin and Johnson [6] proposed a simple method of analysis, based upon an elastic-perfectly plastic model, which seemed to describe the experimental surface displacement rates of Dural and copper. However, a subsequent analysis by FEM [3] and a different semi-analytical analysis by Hearle and Johnson [4] of the perfectly plastic ratchet rate largely overestimated (a factor 5 or so) the experimental rates as shown in Fig. 1 together with some earlier test data of Hamilton [7]. Also included in the figure is the original Merwin's perfectly plastic solution which seemed to fit the experiments well (in contrast with the prediction of Barghava et al. [3], line B). Finally, also included in the figure is the much more refined Bower and Johnson
[8] non-linear hardening solution based on a kinematic hardening model (line $\mathrm{C}$ ) which yet does not show the agreement with experiments Merwin seemed to find with a so much simpler model.

Although research is still very much active on complex hardening models and their ability to predict ratchetting, and a detailed account is not the scope of the present note. It should suffice to say that, regardless on the complexity of the model, the long term ratchetting behaviour which is typically measured in rolling contact experiments is very difficult to predict from constitutive models calibrated on uniaxial or biaxial experiments, simply because such experiments generally run for only few hundreds of cycles at the strain levels needed for ratchetting, so that the measured plastic behaviour in the two experiments.

Turning back on the theme of this note, Fig. 2 returns to the original figure in Merwin's thesis (corresponding to the lower part of Fig. 1) which includes the mild steel, copper and Dural data. The uniaxial yield stresses taken by Merwin are indicated on the uniaxial stress-strain curves for the three materials in Figs. 3-5. For steel and Dural the value corresponds to the distinct initial yield point for steel and about the $1 \%$ proof stress for Dural. However, for copper, Merwin chose a value of $\sigma_{\mathrm{y}}=30,000 \mathrm{psi}$ which corresponds to $25 \%$ deformation, i.e. nearly the ultimate stress for a linear strain measure. A more reasonable assumption would bring to use a value of $\sigma_{\mathrm{y}}=13,000 \mathrm{psi}$

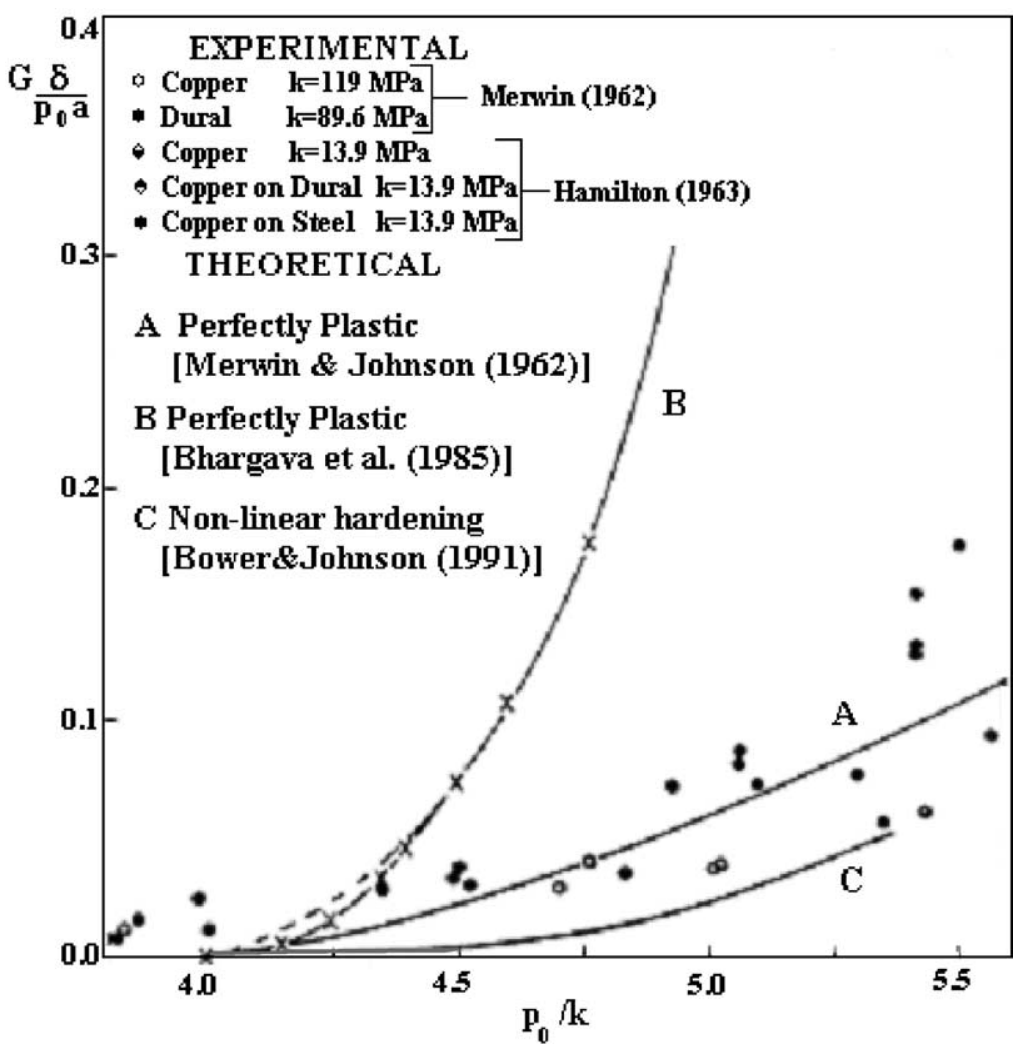

Fig. 1. Steady-state surface displacement per cycle in pure rolling. 


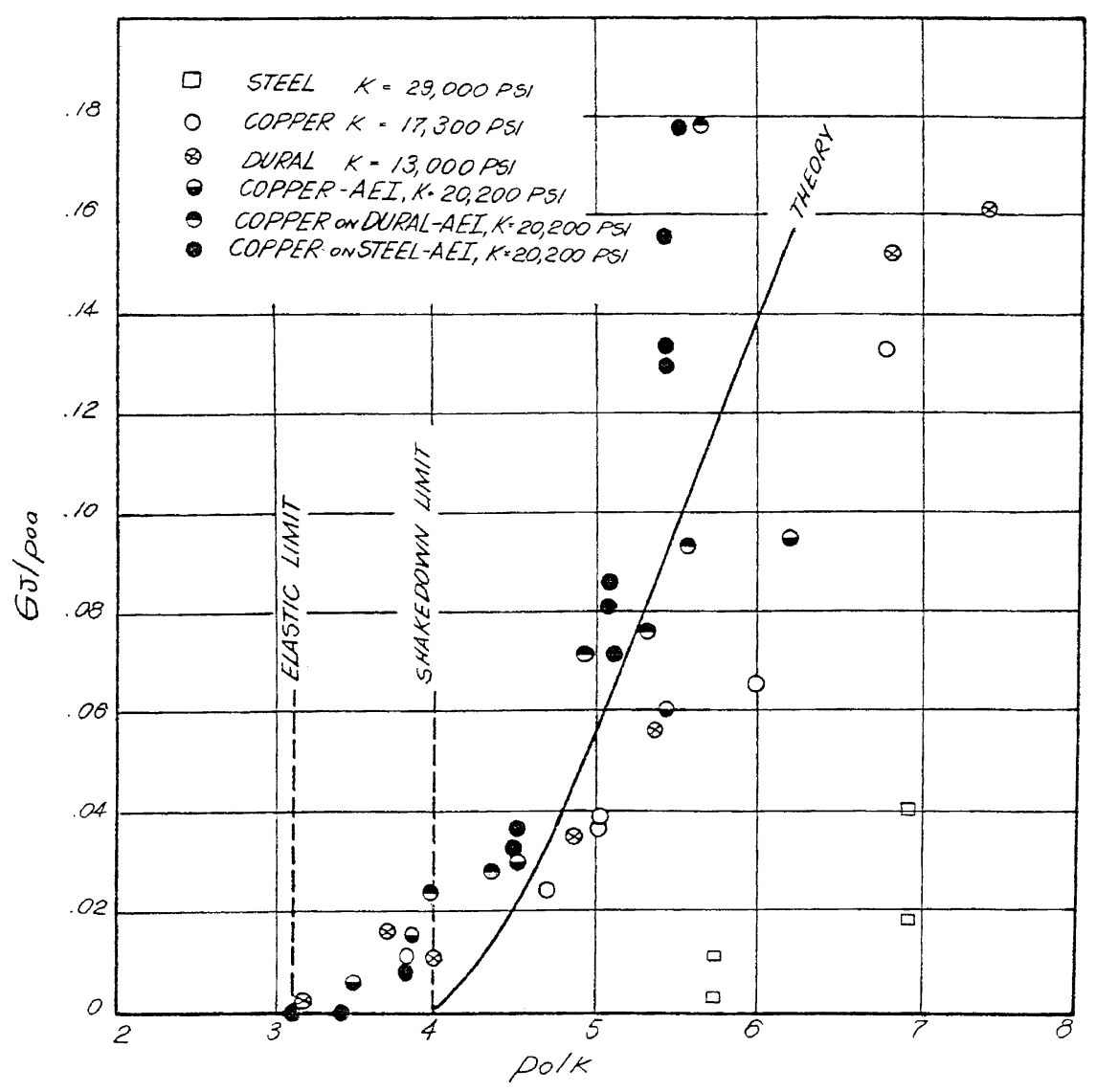

Fig. 2. Tangential displacement versus load factor [2].

which corresponds to the $1 \%$ proof stress. However, with such a value of yielding limit, the copper ratchet rates corresponding to $p_{0} / k_{0}=4$ in Fig. 2 would be transposed to $p_{0} / k_{0}=9.2$ placing the copper data well to the right of the region of the diagram occupied by the steel data.

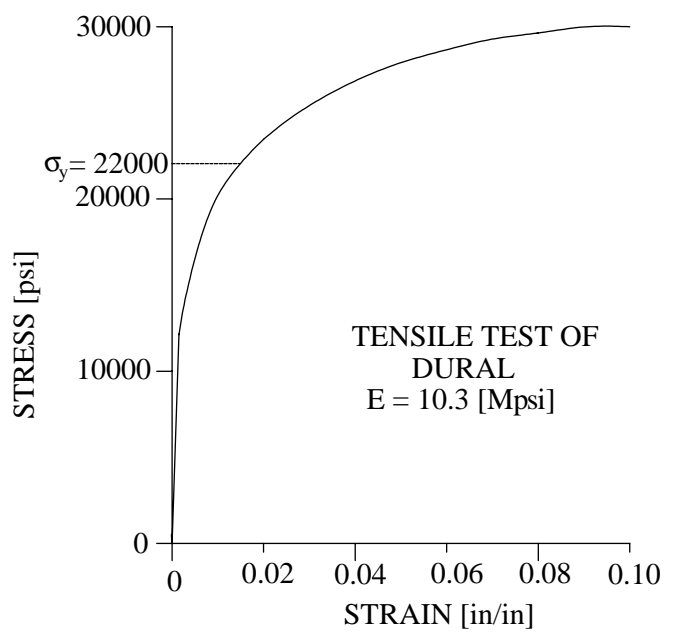

Fig. 3. Tensile stress-strain curve for Dural [2].

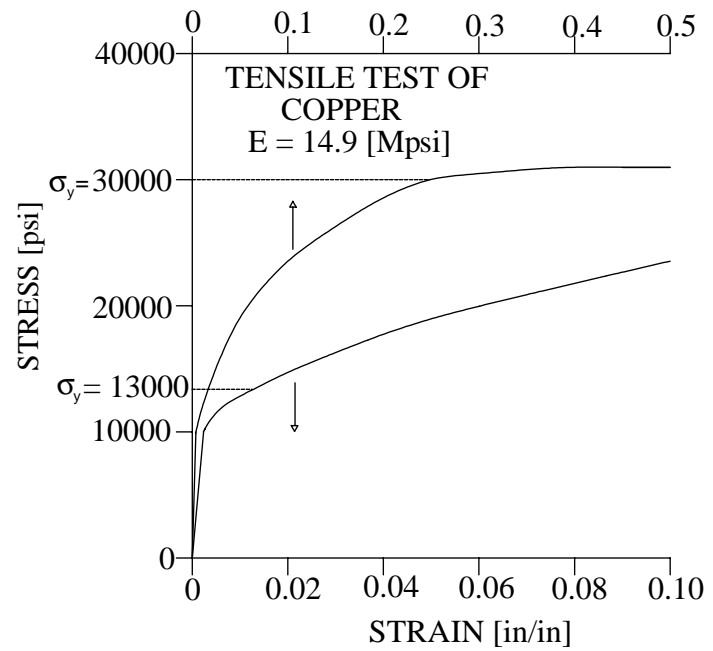

Fig. 4. Tensile stress-strain curve for copper [2].

\section{Structural and material ratchetting (MR)}

Although the observed perfectly plastic ratchet rate overestimates the observed rates, as shown in Fig. 1, the Merwin's data for the three materials lie within an order of magnitude of these rates. Fig. 2 underlines that the primary 


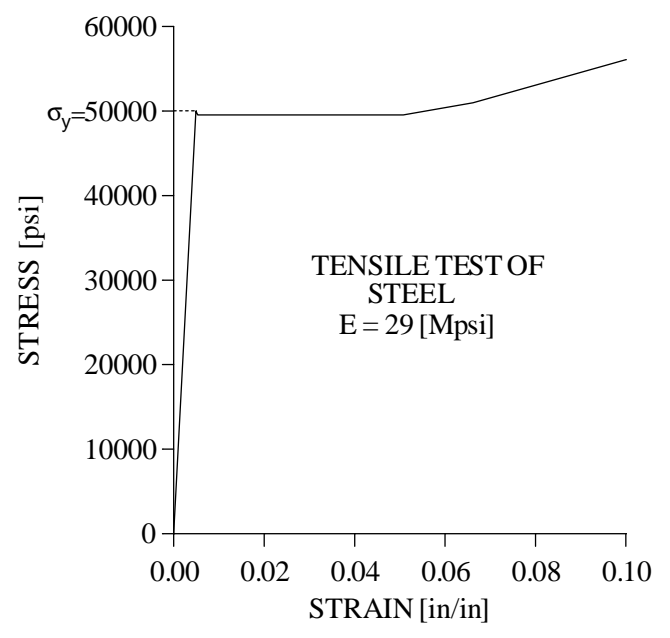

Fig. 5. Tensile stress-strain curve for mild steel [2].

difference between the material responses is the level of load at which ratchetting begins. Given the data seem to fit quite well with the given choice of parameter groups in the coordinate axes $\left(p_{0} / k\right.$, and $\left.G \delta / p_{0} a\right)$, the data gives the impression that "similar" deformation processes are taking place in each material, that the ratchet rate corresponds to a proportion of the elastic strain corresponding to a significant stress level which governs the perfectly plastic ratchet rate where the significant stress level relates to the excess above the shakedown limit. However, the load, hence the stress level, at which the process is initiated varies significantly between the materials.

These features are characteristic of MR which rules the steady cyclic growth of strain which occurs for repeated cycles of stress and that it is observed in the copper and aluminium. Further, the ratchetting in steels only occurs if the maximum stress is significantly above initial yield and approaches the ultimate yield stress. Therefore, the Merwin data are consistent with these characteristics except with the high value of $p_{0} / k_{0}$ for copper: in fact, $p_{0} / k_{0}=9.2$ is much higher than what would be anticipated. However, of the three materials, copper is the only one which exhibits significant cyclic strain hardening. A comparison between the monotonic and cyclic curves for $99.9 \%$ pure copper are shown in Fig. 6 and it can be seen that the cyclic yield value is $2-3$ times the monotonic yield value. Hence, cyclic hardening may explain the relative resistance to ratchetting in the copper tests.

Therefore, although it is not a rigorous solution to the plasticity problem, we can assume a transient process such that the structural ratchetting (SR) (the ratchetting predicted by the Hearle and Johnson model [4]) governs the transient regime regulated by the "distance" from the instantaneous yield limit. Assuming the yield limit will increase with the deformation flow, and for simplicity we can take an estimate from the monotonic tensile curve. This process will clearly rapidly lead to the steady state, after a certain low

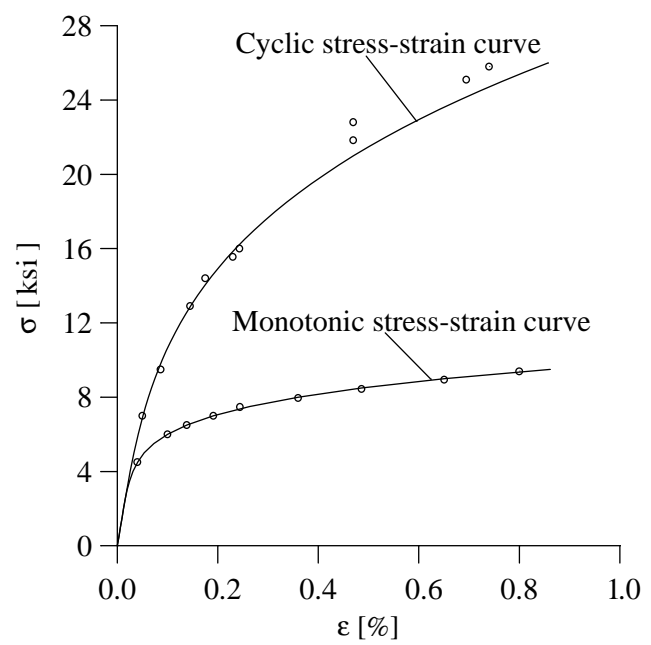

Fig. 6. Monotonic and cyclic stress-strain curves for $99.9 \%$ pure copper [9].

number of cycles, and the following process can be more closely attributed to MR of the hardened structure of the material.

This model does not correspond to any mathematically correct plasticity model, neither isotropic nor kinematic hardening, but will give an estimate on the number of cycles where the transient effect of the cyclic increase of yield limit is affecting the response of the material (SR), with respect to the regime (MR). Obviously, modern non-linear kinematic hardening models obtain the ratchetting response from the dynamic recovery term (back-stress) regulating the movement of the hardening surface. In this respect, SR is the ratchetting occurring for a mechanism of (progressive) collapse. With simple hardening models, this mechanism is resisted so well that ratchetting disappears (isotropic or linear kinematic hardening), unless a sophisticated treatment is calibrated so that MR is predicted based because of the transient response over the cycle of deformation. However, this modelling of MR is not very efficient, because the initial response is not very relevant in the typically rolling contact experiment, and only the steady-state response is of interest.

\subsection{Transient ratchet rates}

The Hearle and Johnson solution [4], HJ in the following, predicts the following ratchet rate (best fit from numerical results in the range $4 \leq p_{0} / k_{0} \leq 4.75$ )

$\frac{G}{a p_{0}} \frac{\mathrm{d} \delta}{\mathrm{d} N}=0.304\left(\frac{p_{0}}{k_{0}}-4\right)^{2}$

Assuming that deformation occurs over a depth of about $0.75 a$ beneath the surface, like in the HJ perfectly plastic solution, we obtain

$\frac{G}{p_{0}} \frac{h}{a} \frac{\mathrm{d} \gamma}{\mathrm{d} N}=0.304\left(\frac{p_{0}}{k_{0}}-4\right)^{2}$ 


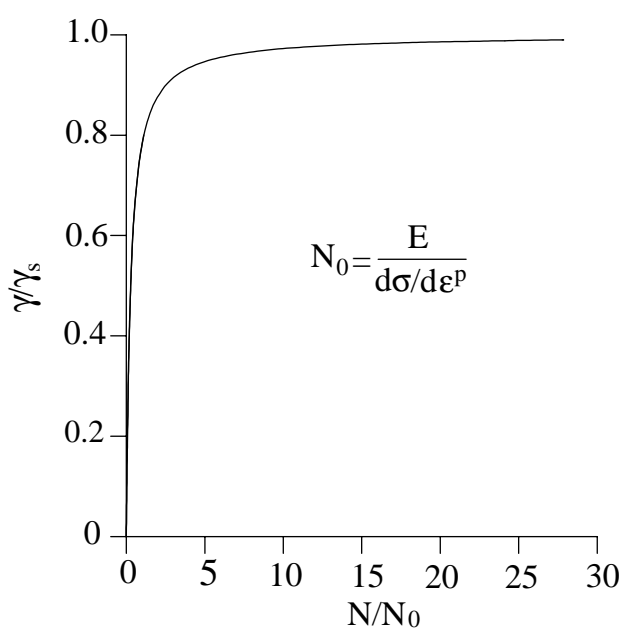

Fig. 7. Eq. (6) for copper and for $p_{0} / k_{0}=4.7$ and $h=0.75 a$.

We know suppose a simple linear hardening law of the type

$k(\gamma)=k_{0}(1+\chi \gamma)$

where $\chi$ is the hardening coefficient, and clearly by differentiating

$\chi=\frac{1}{k_{0}} \frac{\mathrm{d} k(\gamma)}{\mathrm{d} \gamma}$

By substituting for $k$ in the $\mathrm{HJ}$ equation, we get

$\frac{G}{p_{0}} \frac{h}{a} \frac{\mathrm{d} \gamma}{\mathrm{d} N}=0.304\left(\frac{p_{0}}{k_{0}(1+\chi \gamma)}-4\right)^{2}$

Therefore, the number of cycles to a saturated state can be calculated in the following way:

$$
\begin{aligned}
\frac{N(\gamma)}{N_{0}}=0.6\{ & {\left[\frac{\left(p_{0} / k_{0}\right)^{2}+\left(\left(p_{0} / k_{0}\right)-4\right)^{2}\left(1-\gamma / \gamma_{\mathrm{s}}\right)}{64\left(\left(p_{0} / k_{0}\right)-4\right)\left(1-\gamma / \gamma_{\mathrm{s}}\right)}\right] \frac{\gamma}{\gamma_{\mathrm{s}}} } \\
& \left.+\frac{p_{0}}{32 k_{0}} \ln \left|1-\frac{\gamma}{\gamma_{\mathrm{s}}}\right|\right\}
\end{aligned}
$$

where $N_{0}=E /\left(\mathrm{d} \sigma / \mathrm{d} \varepsilon^{\mathrm{p}}\right)\left(E\right.$ is the Young's modulus, $\mathrm{d} \sigma / \mathrm{d} \varepsilon^{\mathrm{p}}$ the slope of the uniaxial stress-plastic strain curve) and $\gamma_{\mathrm{s}}$ the saturation strain $(\mathrm{d} \gamma / \mathrm{d} N=0)$

$\gamma_{\mathrm{s}}=\frac{1}{\chi}\left(\frac{p_{0}}{4 k_{0}}-1\right)$

From the uniaxial stress-strain curves, the average straining hardening over the relevant stress range is

$N_{0}= \begin{cases}80 & \text { for copper } \\ 20 & \text { for Dural }\end{cases}$

Fig. 7, where Eq. (6) is plotted for $p_{0} / k_{0}=4.75$, shows that the transient regime has practically ceased when $N_{t}=5 N_{0}$. Solutions for other values of $p_{0} / k_{0}$ produce similar values. Therefore, keeping in mind the equality (4), the transient strain growth ceases when $N_{t}=400$ cycles for copper and

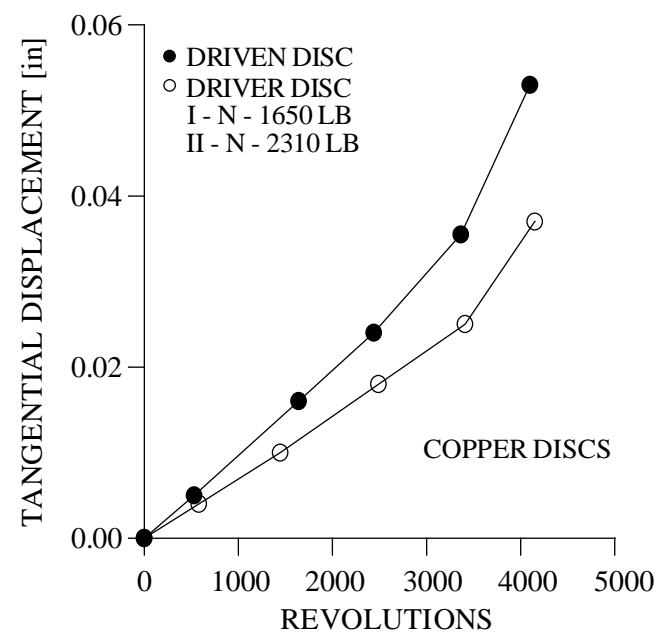

Fig. 8. Surface displacement versus number of revolutions for copper [2].

$N_{t}=100$ cycles for Dural. Figs. 8 and 9 show that the first measurement of ratchet rate in Merwin's tests were made at 500 cycles, when the transient behaviour would have ceased. Thus, the subsequent near constant growth of strain for cycle is MR and its value is unrelated to the predictions of perfect plasticity. This justifies the discrepancy between the experimental Merwin's data and the elastic-perfectly plastic solutions of Hearle and Johnson [4] and Barghava et al. [3].

\subsection{Steady-state ratchet rate: $M R$ in copper}

There are three sets of material ratchet tests for copper available in the literature [9-11]. All the tests were of similar form, a uniaxial stress history cycled between a maximum stress $\sigma_{\max }$ and $\sigma_{\min }=\sigma_{\max }-\Delta \sigma$, where $\Delta \sigma$ is the stress variation.

By plotting the strain-cycle curve (Fig. 10), it can be observed that such curve is analogous to the strain-time curves obtained in conventional creep testing. Beginning from such

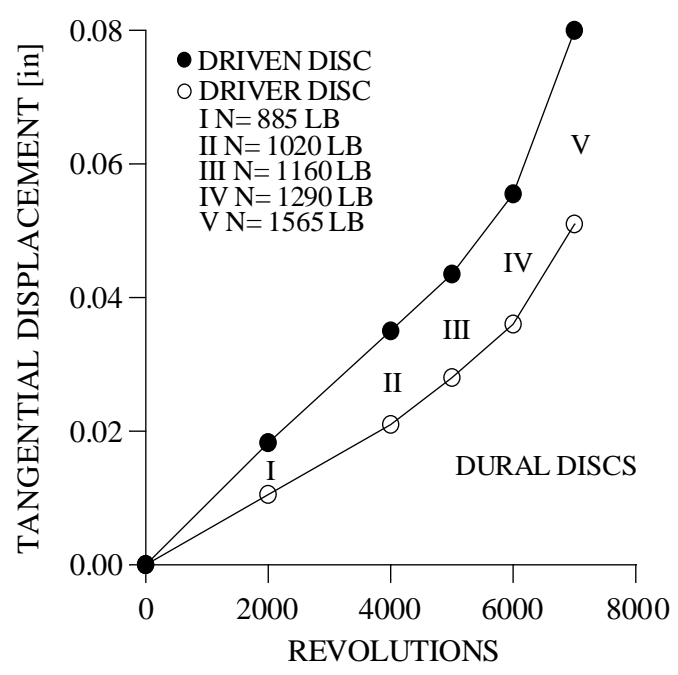

Fig. 9. Surface displacement versus number of revolutions for Dural [2]. 


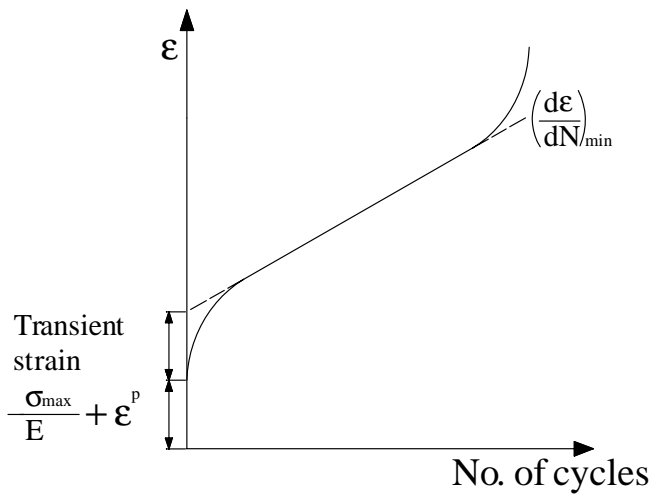

Fig. 10. The strain-cycle curve [9].

observation $[9,10]$ the steady-state ratchet rate has been modelled by power-law expression (a creep-analogue):

$$
\frac{\mathrm{d} \varepsilon}{\mathrm{d} N}=K \sigma_{\max }^{\alpha} \Delta \sigma^{\beta}
$$

where the coefficients $\alpha, \beta$ and $K$ are function of the material properties, but also the stress conditions. Their values, together with the stress ranges and material specification are given in Table 1. It can be seen that the tests were conducted over differing stress ranges and the initial states of the copper differed. The stress histories which occur in the Merwin tests involve multiaxial states of stress, although dominated by shear deformation, and a cyclic state where the variation of stress is symmetrical, i.e. $\Delta \sigma \cong 2 \sigma_{\max }$. Therefore, the use of Eq. (9), where the stress is uniaxial and the variation of stress is less than the maximum stress implies an extrapolation which may not be justified. Further, the copper in the Merwin tests is likely to have experienced cyclic hardening before the steady ratchet rate was reached. Of the three sets of tests, the most extensive and the stress ranges which most closely approximates those which occur in the test is provided by the wire tests. By adopting the Von Mises equivalent quantities, Eq. (9) can be rewritten in the following form:

$\frac{\mathrm{d} \gamma}{\mathrm{d} N}=(\sqrt{3})^{\alpha+\beta+1} K \tau_{\max }^{\alpha} \Delta \tau^{\beta}$

where $\tau_{\max }$ is the maximum elastic shear stress in the direction $z$, perpendicular to the rolled surface which occurs during the cycle. The maximum shear stress $\tau_{\max }$ can be calculated beginning from the McEwen relation [12]

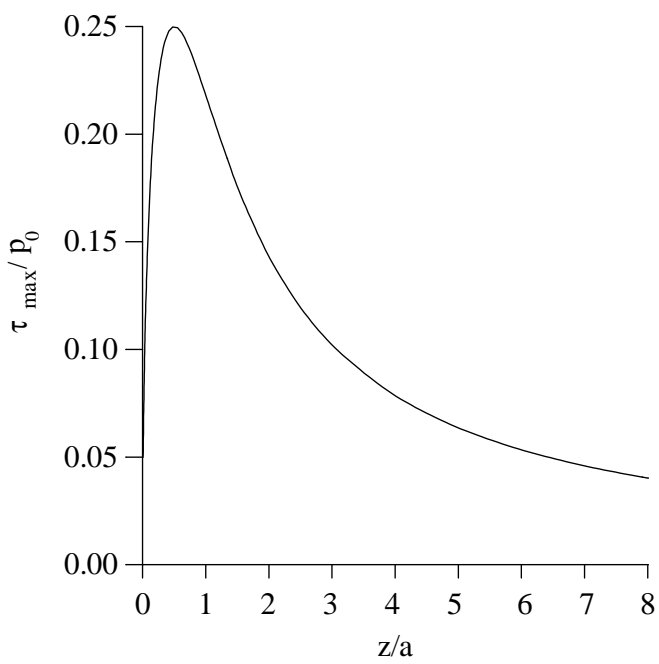

Fig. 11. Variation of $\tau_{\max } / p_{0}$ with the dimensionless depth $z / a$.

$\tau_{x z}=\frac{p_{0}}{a} n\left(\frac{m^{2}-z^{2}}{m^{2}+n^{2}}\right)$

with

$m^{2}=\frac{1}{2}\left\{\left[\left(a^{2}-x^{2}+z^{2}\right)^{2}+4 x^{2} z^{2}\right]^{1 / 2}+\left(a^{2}-x^{2}+z^{2}\right)\right\}$,

$n^{2}=\frac{1}{2}\left\{\left[\left(a^{2}-x^{2}+z^{2}\right)^{2}+4 x^{2} z^{2}\right]^{1 / 2}-\left(a^{2}-x^{2}+z^{2}\right)\right\}$

and imposing $\partial \tau(x, z) / \partial x=0$. Only for two solutions of the this last equation

$$
\begin{aligned}
& \frac{x}{a}=-\left\{1+\frac{5}{3}\left(\frac{z}{a}\right)^{2}-\frac{2}{3} \frac{z}{a}\left[3+a\left(\frac{z}{a}\right)^{2}\right]^{1 / 2}\right\}^{1 / 2}, \\
& \frac{x}{a}=\left\{1+\frac{5}{3}\left(\frac{z}{a}\right)^{2}-\frac{2}{3} \frac{z}{a}\left[3+a\left(\frac{z}{a}\right)^{2}\right]^{1 / 2}\right\}^{1 / 2}
\end{aligned}
$$

the shear stress is maximum and its expression can be obtained by substituting (13) into (11) and (12). Fig. 11 shows the variation of the $\tau_{\max }$, normalised with respect to the Hertzian pressure $p_{0}$, with the depth $z$, normalised with respect to the contact half-dimension $a$.

For the variation of stress twice the maximum stress, as the increment of surface displacement is then given by

$\delta=\int_{0}^{\infty} \frac{\mathrm{d} \gamma}{\mathrm{d} N} \mathrm{~d} z$

Table 1

Values of the coefficients $\alpha, \beta$ and $K$ together with the stress ranges and material specification

\begin{tabular}{llllc}
\hline Material & $\alpha$ & $\beta$ & $K$ & \multicolumn{2}{c}{$\sigma_{\text {max }}(\mathrm{psi})$} & & \\
\hline $99.9 \%$ pure copper [9] & 3.2 & 0.1 & $1.6 \times 10^{-18}$ & $1-10$ \\
Copper wire [10] & 4.625 & 0.888 & $8.23 \times 10^{-29}$ & $1-10$ \\
$99.99 \%$ pure copper [11] & 18.5 & 5.47 & $5.44 \times 10^{-39}$ & $1.5-7.8$ \\
\hline
\end{tabular}




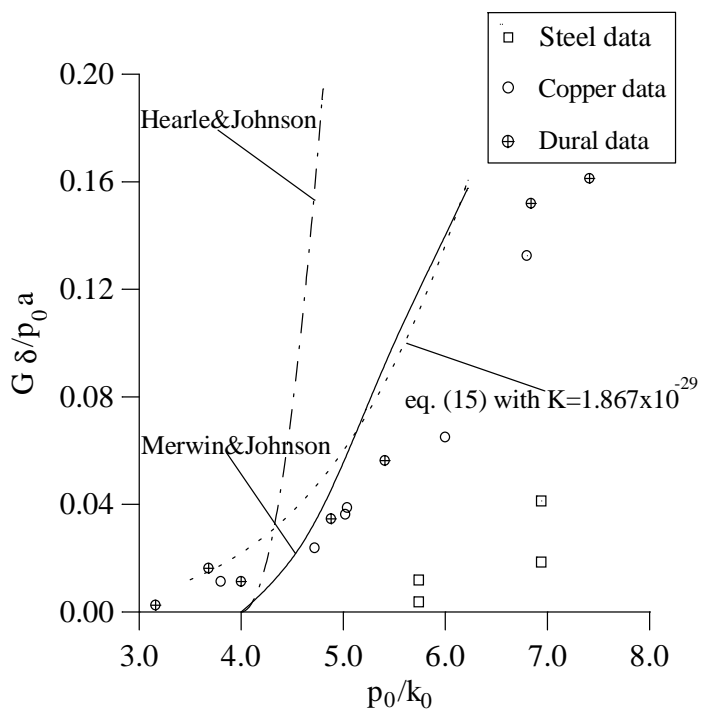

Fig. 12. Dependence of dimensionless ratchet rate on the $p_{0} / k_{0}$.

After a little algebra, (10) can be written in the following form:

$$
\frac{G \delta}{p_{0} a}=B\left(\frac{p_{0}}{k_{0}}\right)^{\alpha+\beta-1}
$$

where the material constant $B$ is given by

$$
B=2^{\beta} \sqrt{3}^{\alpha+\beta+1} K G\left\{\int_{0}^{\infty}\left[\frac{\tau_{\max }}{p_{0}}\left(\frac{z}{a}\right)\right]^{\alpha+\beta} \mathrm{d}\left(\frac{z}{a}\right)\right\} k_{0}^{\alpha+\beta-1}
$$

Numerical integration and substitution of material constants in (16) produces the following value for $B=1.717 \times 10^{-4}$. However, the best fit to the Merwin tests is given by $B=$ $0.42 \times 10^{-4}$, and we will assume that the cyclic hardening in the ratchetting tests justifies this difference. In Fig. 12, the dimensionless ratchet rate $G \delta / p_{0} a$ is plotted as a function of the load level $p_{0} / k_{0}$, as predicted by Eq. (15). In Fig. 12, the elastic-plastic solutions of Hearle and Johnson and Merwin and Johnson are also included, and finally, the power-law curve which gives the best fit to the Merwin tests. It can be observed that the Eq. (15) involves a variation of ratchet rate with $p_{0} / k_{0}$ that closely matches the experimental data. The prediction would have been accurate if the MR equation was given by the values of $\alpha$ and $\beta$ for the wire tests but with $K=1.867 \times 10^{-29}$, corresponding to the best fit $B=0.42 \times 10^{-4}$. However, the reduction of a factor 4 of the ratchet rate suggests, again, that uniaxial data are, once again, not reliable to make quantitative estimates. This calculation is therefore only of qualitative significance. It is actually to remark that an alternative choice of the data from Table 1 would lead not only to loss of correlation of the factor in the power-law curve, but actually to a largely different power-law expo- nent $(\alpha+\beta=12$ and 2.3, respectively, for the other material data in Table 1). In the lack of precise experimental data on the same material, as well as on other conditions and materials, very little is known of MR phenomenon under RCF conditions. Kapoor [13] has suggested then that the condition of failure under RCF ratchetting would essentially be ductile failure (exhaustion of strain ductility), but even this critical condition would require further investigation for its dependence on stress and material conditions.

\section{Conclusions}

In the present note the classical Merwin results for ratchet rates on rolling contact tests have been re-examined. It has been shown that Merwin measured only the long-term behaviour of the plastic flow, which for copper is a steady-state ratchet rate having very little to do with the SR predicted by a perfectly plastic model, and is typical of MR on the hardened structure. A simple calculation is shown here using a 1D calculation and a Megahed "creep-analogue" ratchetting empirical equation. Using material data from similar materials and uniaxial loading condition, a qualitative agreement is found, although the method is not expected to lead to general and quantitative accuracy. Further knowledge and experimental evidence are required both for prediction of the long term ratchetting phenomenon, and for predicting the critical amount of ratchetting which can be tolerated for a given material in a given RCF condition. Nearly 40 years after the apparent success of Merwin's interpretation of his ratchetting experiments, many open questions remain on this phenomenon.

\section{References}

[1] Crook, Simulated gear-tooth contacts: some experiments upon their lubrication and subsurface deformation, Proc. Inst. Mech. Eng. London 171 (1957) 187.

[2] J.E. Merwin, Plastic deformation of surfaces in rolling, Ph.D. Dissertation, Cambridge University, UK, 1962.

[3] V. Barghava, G.T. Hann, C.A. Rubin, An elastic-plastic finite-element model of rolling contact. Part I. Single contacts, J. Appl. Mech., Trans. ASME 52 (1985) 67-74;

V. Barghava, G.T. Hann, C.A. Rubin, An elastic-plastic finite-element model of rolling contact. Part II. Repeated contacts, J. Appl. Mech., Trans. ASME 52 (1985) 75-82.

[4] A.D. Hearle, K.L. Johnson, Cumulative plastic flow in rolling and sliding line contact, J. Mech. Phys. Solids 33 (1987) 61.

[5] R.A. Smith, Fatigue in transport: problems, solutions and future threats, Process Safe Environ. 76 (B3) (1998) 217-223.

[6] J.E. Merwin, K.L. Johnson, An analysis of plastic deformation in rolling contact, Proc. Inst. Mech. Eng. 177 (1963) 676.

[7] G.M. Hamilton, Plastic flow of rollers loaded above their yield point, Proc. Inst. Mech. Eng. 177 (1963) 667.

[8] A.F. Bower, K.L. Johnson, The influence of strain-hardening on cumulative plastic-deformation in rolling and sliding line contact, J. Mech. Phys. Solids 37 (4) (1989) 471. 
[9] M. Megahed, A.R.S. Ponter, C.J. Morrison, A theoretical and experimental investigation of material ratchetting rates in a bree beam element, Int. J. Mech. Sci. 25 (12) (1983) 917-933.

[10] M. Megahed, A.R.S. Ponter, C.J. Morrison, Experimental investigations into the influence of cyclic phenomena of metals on structural ratchetting behavior, Int. J. Mech. Sci. 26 (11/1) (1984) 625-638.

[11] C.E. Feltner, G.M. Sinclair, Cyclic stress induced creep of close-packed metals, in: Proceedings of the Joint International
Conference on Creep, vol. 178, Part 3A, Paper 7, Institute of Engineering, 1963.

[12] K.L. Johnson, Contact Mechanics, Cambridge University Press, Cambridge, 1985.

[13] A. Kapoor, A re-evaluation of the life to rupture of ductile metals by cyclic plastic strain, Fatigue Fract. Eng. Mater. Struct. 17 (1994) 201-219. 